Intropica 14(1): 33 - 42

\title{
Estrategias de educación ambiental para promover la conservación del pato de torrente Merganeta armatta colombiana en dos instituciones educativas rurales del municipio de Salento, Quindío, Colombia
}

\section{Environmental education strategies to promote the conservation of torrente duck Merganeta armatta colombiana in two rural educational institutions of the municipality of Salento, Quindío, Colombia}

\author{
Juan Ernesto López-Londoño@ y Álvaro Botero-Botero*®
}

*Autor de correspondencia: abotero@uniquindio.edu.co

Recibido: 30 octubre de 2018

Aceptado: 02 de abril de 2019

Grupo de investigación Biodiversidad y Educación Ambiental - BIOEDUQ Universidad del Quindío, Armenia, Colombia

Palabras clave:

alto Cauca; educación ambiental; educación básica; educación para el desarrollo sostenible; enseñanza de las ciencias

upper Cauca; environmental education; basic education; education for sustainable development; science education

\section{Resumen}

El pato de torrente (Merganetta armata) se encuentra distribuido en la región Andina en todo el continente suramericano. Esta especie presenta requerimiento específico de hábitat, como aguas libres de contaminación, vegetación ribereña abundante y baja presencia de humanos, lo que lo hace altamente vulnerable a los impactos antrópicos. Esto hace que las comunidades ribereñas cumplan un papel importante en la conservación de la especie y de los ecosistemas ribereños. Por ello, se diseñó e implementó una estrategia de educación ambiental para promover la conservación del pato de torrente M. armata colombiana en dos sedes rurales de la Institución Educativa Boquía, en el municipio de Salento (Quindío). Se trabajó con niños y niñas hijos de campesinos de las veredas Cocora y Palo Grande, donde se realizó un diagnóstico previo sobre los conocimientos básicos de los niños acerca de la especie. Se encontró que, en general, los niños desconocían el pato de torrente y solo estaban familiarizados con el estereotipo de los patos asociados a lagos. A partir de dicho diagnóstico, se diseñó una cartilla base de trabajo sobre diversos aspectos de la ecología, la biología y la conservación del pato de torrente, la cual acompañó la realización de cuatro jornadas en cada centro educativo. Asociado a esto, se implementaron como refuerzos educativos carteleras, infografías y pato-desafíos (juegos). Al evaluar la estrategia, se concluyó que los niños reconocieron la especie, su ecología y sus amenazas. Solo se presentó dificultad en la comprensión de la dieta de la especie.

\section{Abstract}

The torrent duck (Merganetta armata), inhabit the Andes, in South America. This species needs specific habitat requirements such as pollution-free water, abundant riverine vegetation and low human presence, making torrent duck highly vulnerable to anthropic impacts. This causes riparian communities to play an important role in the conservation of species and ecosystems. Therefore, an environmental education strategy was designed and implemented to promote the conservation of torrent duck in two (2) rural facilities of the Institución Educativa Boquía, in the municipality of Salento (Quindío). The work was developed with children of peasants from the villages of Cócora and Palo Grande, where a preliminary diagnosis was made on the basic knowledge of the children about the species, finding that most of the children were unaware of the duck and they were only familiar with the stereotype of ducks associated with lakes. Based on this diagnosis, a work primer was designed on various aspects of the ecology, biology and conservation of the duck. This primer supported the realization of four workdays in each educational center. In addition, billboards, infographics and duck challenges (games) were implemented as educational reinforcements. When evaluating the strategy, it was concluded that the children recognized the species, its ecology and its threats but they had difficulty in understanding the diet of the species. 


\section{Introducción}

Los patos, los gansos y los cisnes pertenecen a la familia Anatidae y son aves que se encuentran en ambientes fluviales. Sólo cinco de estas especies en sistemas acuáticos lóticos (ríos y quebradas); las demás se encuentran en ecosistemas lénticos (Madge y Burn, 1988). El pato de torrente Merganetta armata se distribuye en Suramérica, y dentro de esta zona es clasificado en las siguientes subespecies: M. armata colombiana, en Venezuela, Colombia y norte de Ecuador; $M$. armata leucogenis, en Ecuador y Perú; M. armata turneri, en Perú; M. armata garleppi, en Bolivia; $M$. armata fraenata, en Chile; $M$. armata armata, en Argentina y Chile, y M. armata berlepschi, en Argentina (Fjeldså y Krabbe, 1990; Carboneras, 1992).

La subespecie M. armata colombiana se distribuye en los ríos y quebradas andinas de Venezuela, Colombia y Ecuador. En Colombia se encuentra a lo largo de los tres ramales de la cordillera (Occidental, Central y Oriental), entre 1467 y 3500 m (Hilty y Brown, 2001; Carboneras, 1992; Ramírez et al., 2014). Estas aves se hallan asociadas exclusivamente a los ríos de corriente rápida de alta montaña (Carboneras, 1992; Naranjo y Ávila, 2003; Cerón et al., 2010), como en el caso del río Quindío, donde se distribuyen desde la zona alta (sector de Cócora, Salento) hasta $1415 \mathrm{~m}$ en el sector de Chaguala, municipio de Armenia (Ramírez et al., 2014).

Los patos de torrentes son aves vistosas y se caracterizan por tener cabeza y cuello blancos con líneas negras, dorso negruzco con estrías claras, pico rojo y cola ancha, larga y rígida (en el macho); las hembras presentan corona y cuello grises, y las partes ventrales son de color canela (Carbonell et al., 2007) La especie forma parejas monógamas permanentes que defienden un mismo territorio durante todo el año (Moffett, 1970).

Merganetta armata se ve afectada por factores naturales y antrópicos, como son el turismo poco planificado, la pérdida de vegetación ribereña, la contaminación de las fuentes hídricas, la expansión de la frontera agrícola y los asentamientos humanos cercanos al cauce (Ramírez et al., 2014). Estas características son propias del lugar donde se realizó el presente trabajo; de allí la necesidad de involucrar a la comunidad aledaña a través de la Institución Educativa Boquía y sus sedes alternas, para lograr el reconocimiento y apropiación de la especie por parte de los lugareños y su participación activa en el cuidado de sus hábitats. Con este fin, se implementó una estrategia de educación ambiental para promover la conservación del pato de torrente en dos sedes rurales de la Institución Educativa Boquía, ubicada 34 en el municipio de Salento (Quindío, Colombia).

\section{Materiales y métodos}

\section{Área de estudio}

El municipio de Salento se encuentra situado en el sector nororiental del Quindío, Colombia, sobre el flanco occidental de la cordillera central; presenta alturas que van desde los $1300 \mathrm{msnm}$ hasta los $4750 \mathrm{msnm}$, en los farallones nevados del Quindío. El municipio es irrigado por el río Quindío, el cual nace al noreste del departamento, en la confluencia de las Quebradas Cárdenas y San José, en la vereda Cócora. Dicho afluente recorre de norte a sur el departamento hasta el valle de Maravélez, donde, junto con el río Barragán, forman el río La Vieja en límites con el departamento del Valle del Cauca (Botero-Botero et al., 2016). En su recorrido, el río Quindío tiene una longitud de $58,56 \mathrm{~km}$ y abastece los acueductos municipales de Armenia, Circasia, Salento y La Tebaida (Corporación Autónoma Regional del Quindío, 2008).

En el municipio de Salento se seleccionaron para el presente trabajo dos sedes de la Institución Educativa Boquía, el centro docente (C.D.) "República de Colombia”, ubicado en la vereda Palo Grande, y el C.D. "San Gabriel”, localizado en la vereda Cócora. Boquía implementa un modelo pedagógico denominado Escuela Nueva, que consiste en convertir al niño en el centro del proceso de enseñanza y aprendizaje, mientras que el profesor deja de ser el punto de referencia fundamental para convertirse en un dinamizador de la vida en el aula, al servicio de los intereses y necesidades de los estudiantes (Villar, 1996). Este modelo, es dirigido a centros educativos multigrados a cargo de un solo docente y creado para las zonas rurales de Colombia (Ministerio de Educación Nacional de Colombia, 2010), es flexible e incluyente, con una pedagogía activa autoestructurante. En la institución también se aplica el Proyecto Ambiental Escolar (PRAE), el cual se fundamenta en el uso, conservación y manejo de los recursos naturales. Vale destacar que la institución está ubicada en la zona de distribución del pato de torrente en el río Quindío. Se trabajó con todos los niños presentes en los dos centros educativos: 12 en el C.D. República de Colombia y 16 en el C.D. San Gabriel. Ellos son hijos de campesinos de la zona, que heredarán el oficio o los predios, por lo cual se consideran actores claves en la conservación de la zona.

\section{Diseño e implementación de la estrategia}

La estrategia de educación ambiental se realizó en cuatro fases. La fase 1 consistió en el diagnóstico del estado actual del conocimiento de los niños acerca de la especie y la actitud hacia a ella. A partir de esto se diseñaron las actividades por aplicar (fase 2), cada una de las 
cuales fue evaluada en el terreno (fase 3). Con base en esta evaluación, se procedió al ajuste y rediseño de las actividades para el siguiente encuentro (fase 4) (figura 1).

Es de aclarar que, antes de iniciar cualquier actividad con los niños, se realizó una reunión de padres de familia donde se explicaron los pormenores del proyecto y se solicitó la firma del consentimiento informado de acuerdo a las políticas de la Vicerrectoría de Investigaciones de la Universidad del Quindío, siguiendo los formatos establecidos para tal fin. Además, se respetaron los códigos de ética en la investigación etnozoológica planteados por Costa Neto et al. (2009).

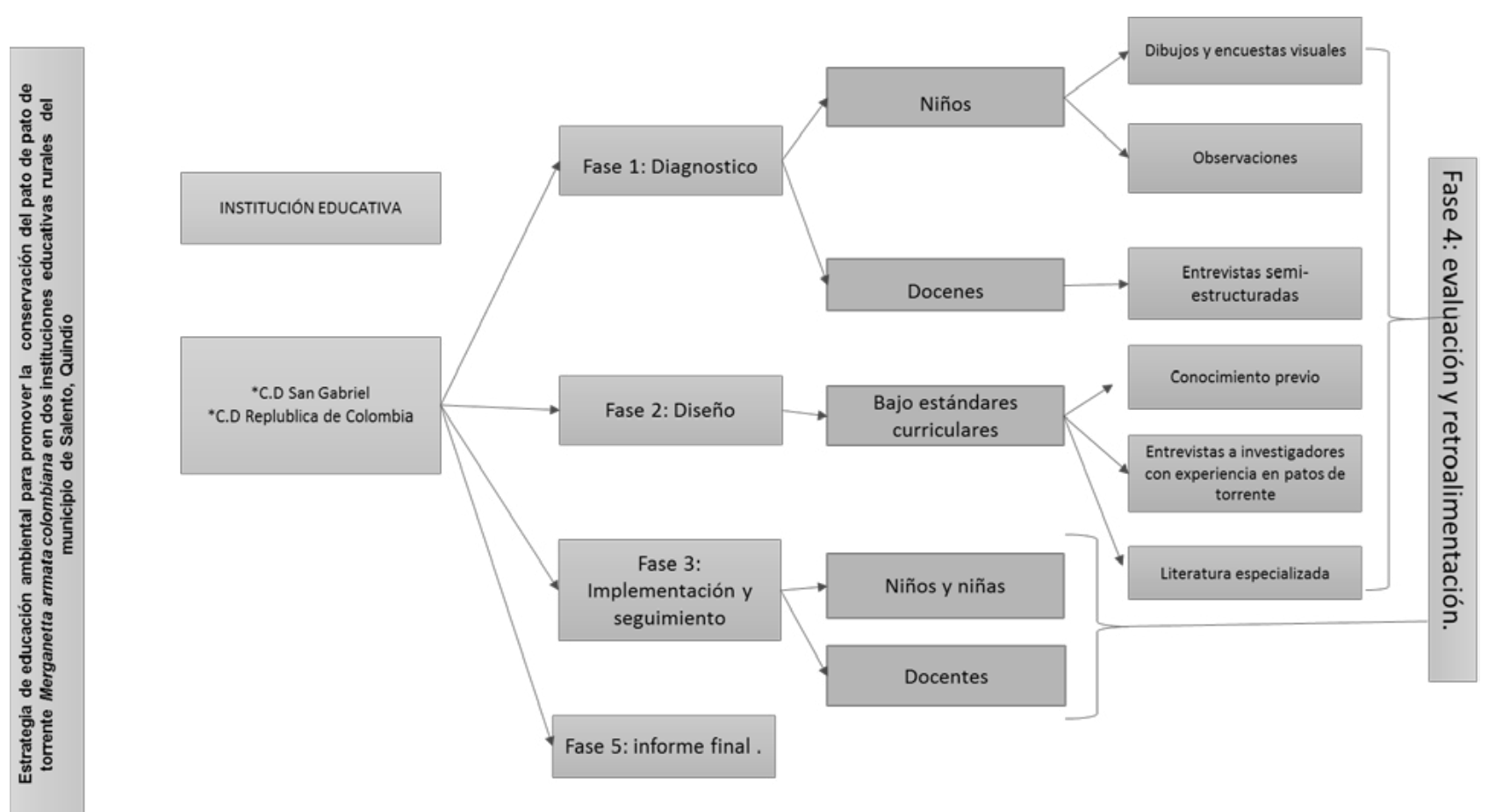

Figura 1. Diagrama de flujo del diagnóstico, diseño, implementación, evaluación y retroalimentación de la estrategia de educación ambiental.

\section{Descripción de las fases}

\section{Fase 1. Diagnóstico}

Se emplearon los dibujos como herramienta metodológica para los conocimientos etnozoológicos de los estudiantes. Se les solicitó a los niños que elaboraran dibujos sobre los patos del río, y para esto se les facilitaron lápices, colores y hojas de papel; una vez terminados dichos dibujos se les pidieron a los niños sus explicaciones, esperando, como lo plantea Santos-Baptista (2009), que mediante estas representaciones gráficas los estudiantes demostraran conocimiento sobre la morfología, la ecología y el comportamiento del animal, así como sobre su interrelación con otros seres vivos. Los dibujos fueron revisados y clasificados según dos criterios, así: el ave (pato) está dibujado en un lago, un río o un ecosistema terrestre; la forma del ave es un pato, un cisne u otra ave. A partir de estos dos tipos de categorías, se aplicó estadística descriptiva. En lo que respecta a los docentes, se les realizó una entrevista semi-estructurada para evaluar el conocimiento sobre la historia natural del pato, los sitios de avistamiento, la presencia de nidos y las amenazas a los mismos.

\section{Fase 2. Diseño}

A partir de los datos obtenidos en la etapa 1 de diagnóstico, se seleccionaron los temas que presentaron mayor debilidad y los que necesitaban reforzamiento con miras a buscar las mejores estrategias didácticas por implementar y el mejor modelo pedagógico que cabía seguir. Además, se esperaba que las estrategias fueran validadas previamente por expertos en la materia antes de ser implementadas. El modelo pedagógico seguido fue aprendizaje por cambio 
conceptual, donde se identifica los conocimientos previos para luego implementar las estrategias didácticas y posteriormente, evaluar el cambio en los conceptos de los educandos (Ruiz, 2007).

\section{Fase 3. Implementación y seguimiento}

En esta etapa del proyecto se procedió a implementar las estrategias diseñadas en la fase 2. Para esto se contó con la participación de todos los niños de cada escuela y de sus profesores. Por medio de acciones indirectas, también se vinculó a los padres de familia.

\section{Fase 4. Evaluación y retroalimentación}

Se realizó una prueba de evaluación a los niños y se diseñaron un pendón y una infografía que fueron dejados en el colegio como retroalimentación final.

\section{Resultados y discusión}

\section{Diagnóstico de conocimientos previos sobre el pato de torrentes}

Como eje inicial de la estrategia, se evaluaron los conocimientos previos de los niños, pues ellos cuentan con un conjunto de ideas propias con respecto a las temáticas que cargan de subjetividad las observaciones y pueden producir concepciones erróneas (Bachelard, 1976), las cuales deben ser subsanadas en la estrategia para que esta sea considerada exitosa. Al evaluar los saberes previos sobre el pato de torrente en los niños de los dos centros docentes, se evidenció que en general desconocían la especie. En el C.D. San Gabriel el 68,75 \% de los niños dibujaron patos en lagos, y solo unos pocos $(6,25 \%)$ mientras que el resto ubicó el ave en ecosistemas terrestres (25\%). En el C.D. República de Colombia los niños muestran el ave en lagos (22,22 \%), en ríos $(22,22 \%)$ y mayoritariamente en ecosistemas terrestres (55,56 \%) (figura 2 ).

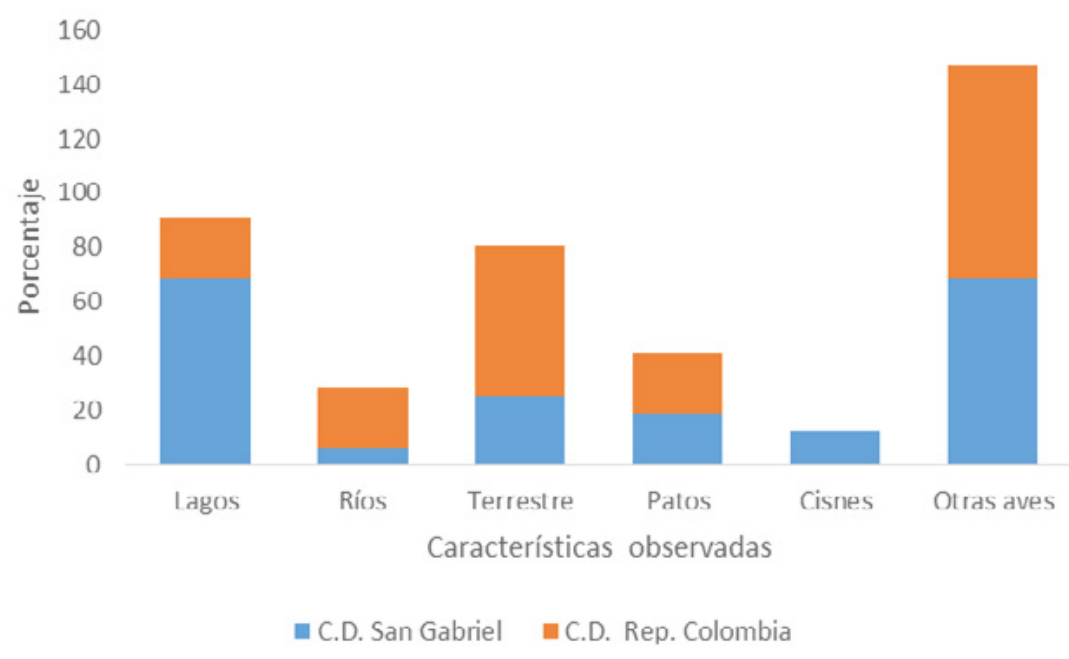

Figura 2. Diagnóstico sobre el conocimiento inicial que presentan los niños en dos centros educativos de la Institución Educativa Boquía (Salento, Quindío) acerca del hábitat y la morfología del pato de torrente.

Los resultados del diagnóstico evidencian que la mayoría de los estudiantes de las dos sedes educativas no conocen el pato de torrente, lo que puede estar asociado a la baja tasa de contacto con la especie, lo que se dificulta por la especialidad de hábitat de esta. Estos organismos viven solo en los cauces del río, hacen poco uso de las orillas (Ramírez et al., 2014) y presentan una distancia mínima de tolerancia hacia las personas de 7,21 m; sin embargo, a partir de los $32 \mathrm{~m}$ y dependiendo de la intensidad de 36 ruido emitido por las personas, los patos ya manifiestan un comportamiento de alerta (Giraldo López y BoteroBotero, 2017), lo cual hace aún más difícil el contacto con la especie. A su vez, los ríos andinos torrentosos como el Quindío suelen ser sitios peligrosos para los niños de corta edad (como los estudiantes de los dos centros docentes), i lo que les impide ir a explorar, jugar o bañarse por sí solos, minimizando así la tasa de encuentro con los patos. A partir de esto, se evidencia la necesidad de reforzar en los niños los siguientes temas: particularidades del hábitat del pato de torrente, el uso que la especie hace del hábitat, su 
dieta, aspectos básicos de su reproducción y su morfología básica, así como acciones humanas que ponen en riesgo su supervivencia.

Este resultado puede estar influenciado por el conocimiento popular de que los patos habitan en lagos, como es común en textos colegiados. En efecto, el $97 \%$ de los patos evidentemente viven en este tipo de hábitat, siendo M. armata una excepción al caso. A nivel mundial, solo cinco de las 148 especies de patos (Anatidae) en el mundo presentan distribuciones restringidas a ríos de montaña (Madge y Burn, 1988). A su vez, se evidencia que los niños de la institución educativa República de Colombia posiblemente han tenido mayor contacto con el pato. En este caso, se evidencia la formación de base de la docente, la cual es licenciada en Biología y Educación Ambiental, además de que en este centro docente se implementaron los talleres diseñados por Botero-Botero et al. (2015). Por otro lado, existen diferencias en los tipos de dibujos realizados por los niños en relación con el tipo de ave: en el C.D. San Gabriel, el 19,75 \% de los niños dibujaron patos; el $12,5 \%$, cisnes, y el $68,75 \%$, otras aves, mientras que en el C.D. República de Colombia aumentó el porcentaje de niños que dibujaron un pato $(22,22 \%)$; el resto $(77,78 \%)$ dibujó otras aves (figura 2).

\section{Diseño}

Para esta fase se planteó como elemento educativo la construcción de una cartilla que consta de 20 páginas, portada y contraportada, con gráficos a color y en blanco y negro, impresa de manera horizontal en hojas de papel de caña de azúcar Reprograf, de color amarillo y de tamaño final $16,5 \times 21 \mathrm{~cm}$. De este documento se generó un depósito digital en la Biblioteca CRAI Euclides Jaramillo Arango, de la Universidad del Quindío. En esta cartilla se incluyeron aspectos básicos de la taxonomía, la morfología y la ecología de la especie y las amenazas de las acciones antrópicas sobre los patos, con base en las deficiencias encontradas en la fase de diagnóstico previo y procurando tener en cuenta la condición multigrada de los centros docentes. Cada capítulo de la cartilla inicia con una pregunta motivacional: “iQuién es el pato de torrente?”, "¿Cómo diferenciamos entre hembras, machos y crías?", “¿En dónde viven los patos?”, “¿Cómo viven?”, “iDe qué se alimentan los patos?”, “¿Cómo es la gestación del pato de torrente?", "¿Qué amenaza al pato de torrente y a su hábitat?”. A su vez, se creó un capítulo asociado a las acciones con las que podemos ayudar a esta especie y a su hábitat, así como un cuento llamado "Anita y Torrentico", basado en el documento de Botero-Botero et al. (2015). Cada capítulo fue tratado en un encuentro con los niños.
Además, se incluyeron un glosario básico y las referencias bibliográficas usadas para la construcción del texto. La cartilla buscó apoyar el cumplimiento de los estándares básicos para la enseñanza de las ciencias naturales (Ministerio de Educación Nacional de Colombia, 2008), en temas relacionados con: proponer y verificar necesidades de los seres vivos; identificar patrones comunes a los seres vivos; explicar adaptaciones de los seres vivos al ambiente; identificar y describir la flora, la fauna, el agua y el suelo del entorno; describir características de seres vivos y objetos inertes; establecer semejanzas y diferencias entre ellos y clasificar; describir y verificar ciclos de vida de seres vivos.

Dentro de la cartilla se encuentran las actividades de refuerzo de cada uno de los temas. Para reforzar los conocimientos adquiridos sobre la morfología del pato, se incluyó una silueta del mismo para ser coloreada al finalizar del tema. Para reforzar el tema de hábitat del pato, la segunda actividad fue un laberinto. Para el refuerzo de los temas de alimentación y hábitat se usó una sopa de letras. Finalmente, para refuerzo general se utilizó un crucigrama.

\section{Implementación}

En esta fase se realizaron cuatro actividades, desarrollando todos los temas de la cartilla.

\section{Primer encuentro}

En esta etapa los niños se reunieron en mesa redonda. A cada estudiante se le entregó una cartilla en la cual se trabajó alrededor de la descripción morfológica del pato y las diferencias entre macho, hembra y pichones. Luego se les pidió que colorearan la silueta del pato.

\section{Segundo encuentro}

En esta actividad se reunieron los niños en mesa redonda y se trabajó la distribución global y local de la especie. Además, se usaron imágenes de apoyo del hábitat y de las subespecies de $M$. armata. Después se desarrolló el primer pato desafío, el cual fue el laberinto impreso en tamaño pliego de cartulina $(70$ x $100 \mathrm{~cm})$ y cuyo objetivo era ayudarle al pato a buscar su hábitat.

\section{Tercer encuentro}

Se reunieron los niños en mesa redonda y se realizó la explicación sobre el tipo de alimentación que tiene el pato de torrente y por medio de diapositivas se les mostraron imágenes de invertebrados acuáticos bentónicos. Luego se les explicaron las características del pico para poder 
alimentarse. Posteriormente, se desarrolló el segundo pato desafío, el cual consistió en una sopa de letras impresa en un pliego de cartulina, donde los niños buscaban las respuestas a las preguntas que se les formulaban.

\section{Cuarto encuentro}

En esta fase se explicó a los niños la gestación del pato, en dónde construyen los nidos, cuánto tiempo se demoran empollando los huevos y hasta qué tiempo cuidan a los pichones. También se les presentó el cortometraje "Biología reproductiva del pato de torrente río Quindío” (disponible en: https://www.youtube. $\mathrm{com} /$ watch $? \mathrm{v}=\mathrm{X} 7 \mathrm{rwfQdJdgE \& t}=6 \mathrm{~s}$ ). Además, se les pregunto a los niños si conocían las amenazas que afectaban a la especie, y muchos de ellos mencionaron de manera intuitiva algunas de ellas. También se les mencionaron algunas acciones que pueden hacer para ayudar a conservar el pato y su hábitat. Entre todos los niños se leyó el cuento de Anita y Torrentico, después se entabló un dialogo sobre el contenido de la historia.. Como refuerzo, se realizó el tercer pato desafío, consistente en llenar un crucigrama en mesa redonda, el cual fue también impreso en un pliego de cartulina y forrado en plástico para ser reutilizado.

\section{Refuerzos educativos dejados en la institución}

Como refuerzo permanente se dejaró en la escuela una cartelera impresa a todo color de $70 \times 100 \mathrm{~cm}$ sobre los patos de torrentes, aliados en la conservación de nuestros ríos, donde se presenta un texto corto sobre la importancia de los patos y nueve acciones que los niños pueden hacer para ayudar a la conservación de la especie. A su vez, se creó una infografía con la información ecológica de la especie, impresa a todo color $(70 \times 100 \mathrm{~cm})$, la cual fue fijada en cada colegio. Antes de ser fijados, estos dos refuerzos fueron socializados con los niños y los docentes.

\section{Evaluación}

Para la evaluación de las actividades se utilizó una prueba de nueve preguntas que contenía ilustraciones para colorear y figuras para encerrar, entre otras modalidades. Este diseño permitió incluir a toda la población estudiantil. Para los niños que no sabían leer, los autores leyeron y explicaron las preguntas, y los alumnos coloreaban las respuestas que consideraban.

Con relación a la pregunta “iDe qué se alimenta el pato?”, en el C.D. San Gabriel el 69 \% de los niños tuvieron tres aciertos; el $15 \%$, dos aciertos, y otro $15 \%$ tuvo un solo acierto. A su vez, el $90 \%$ de los niños del C.D. República de Colombia tuvieron tres aciertos, y el $10 \%$, dos aciertos (figura 3). En este caso, la respuesta correcta fue la selección de tres aciertos, donde se alcanzaron resultados superiores al $60 \%$ en ambas instituciones. El aprendizaje de este tipo de temas se dificulta en razón a que la dieta del pato consta de insectos acuáticos, los cuales se encuentran adheridos a rocas bajo el agua y constituyen formas inmaduras que son pobremente incluidas en los textos de colegio. Además, un posible obstáculo que se ha evidenciado en otros trabajos con población escolar es el asociado a la tendencia general hacia la repulsión de estos organismos, al ser tildados como feos y asquerosos (Robles-Piñeros, 2013).

Frente a la solicitud de señalar con un círculo la manera en que nacen los patos, todos los niños del C.D. San Gabriel acertaron, mientras que en el C.D. República de Colombia el $90 \%$ acertaron y el $10 \%$ fallaron (figura 3). Este es un tema que se puede manejar más fácil con población escolar dado que, a partir de experiencias propias, los niños tienen contacto con la eclosión de un ave a partir de un huevo. Por otro lado, el tema de la reproducción de las aves es ampliamente abarcado en los textos escolares y en las clases de ciencias naturales, pues según los estándares curriculares para la enseñanza de las ciencias naturales desde el grado primero hasta el grado tercero los niños se acercan a este tema al cumplir con el estándar "Describo y verifico ciclos de vida de seres vivos" (Ministerio de Educación Nacional de Colombia, 2008).

Cuando se evaluaron los aspectos básicos de la morfología de los patos, solo el $8 \%$ de los niños del C.D. San Gabriel no acertaron los patrones de coloración; el 69 \% tuvo un acierto, y el $23 \%$ tuvo tres aciertos. El $20 \%$ de los estudiantes del C.D. República de Colombia, por otra parte, no acertó la respuesta correcta, el $10 \%$ tuvo un acierto, y el $70 \%$ tuvo dos aciertos. Respecto a la coloración del pico, el $62 \%$ de los estudiantes del C.D. San Gabriel acertó, mientras que en el C.D. República de Colombia este porcentaje fue del 70 \% (figura 3). Este tipo de pregunta resultó ser muy específica para los niños, lo cual los obligaba a hacer uso de la memoria, y esto dificultó el proceso de evaluación; sin embargo, más del $50 \%$ de ellos retuvieron la información suministrada en el primer encuentro, lo cual es favorable dado el tiempo transcurrido entre la entrega de la información y la evaluación. 


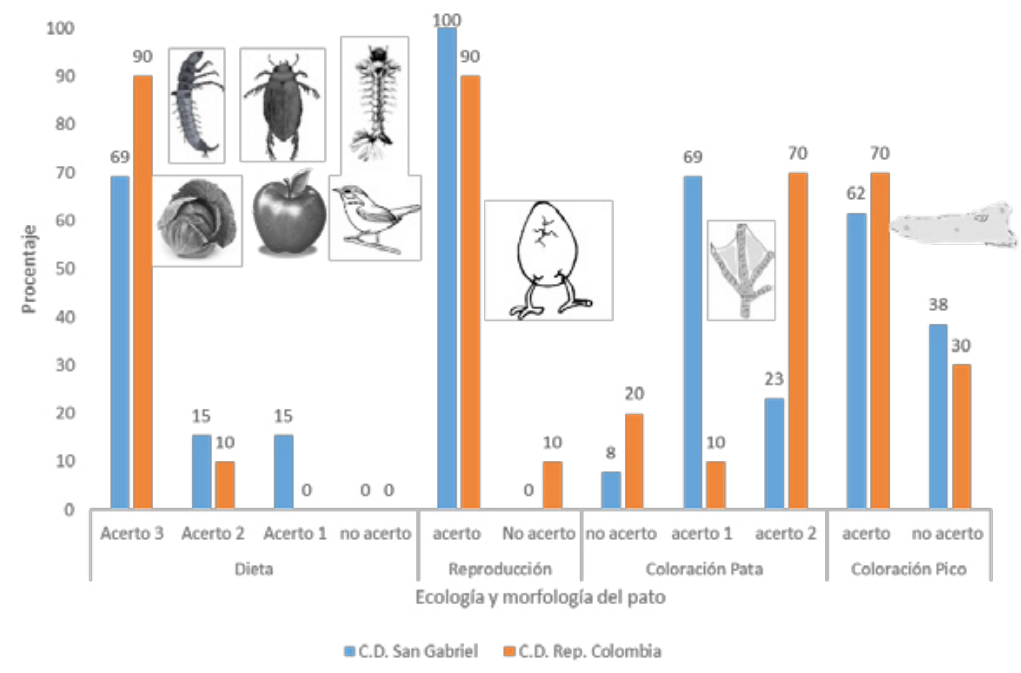

Figura 3. Resultado de la evaluación acerca de los temas de dieta, reproducción y morfología del pato de torrente.

Respecto a las preguntas sobre distribución y hábitat del pato de torrente, el $85 \%$ de los niños del C.D. San Gabriel colorearon de forma acertada los países donde se encuentra la especie; el $15 \%$ restante coloreó otras regiones asociadas a la cuenca del océano Atlántico. En el C.D. República Colombia el $100 \%$ de los estudiantes colorearon de forma acertada los países donde se encuentra la especie. A la solicitud de encerrar el río donde se encuentra la especie en el departamento del Quindío, en el C.D. San Gabriel el 69 \% de los estudiantes acertaron parcialmente y el $31 \%$ acertaron totalmente. En el C.D. República de Colombia el $100 \%$ de los estudiantes acertaron parcialmente (figura 4). En esta última pregunta cabe considerar que en muchos casos la información suministrada a los niños (el mapa de ríos) no les resultó familiar y se les dificultó identificar tanto el departamento del Quindío como el municipio de Salento. Se observó que a los niños les fue más familiar el mapa de América Latina con la división política que el mapa de ríos, posiblemente por la forma de enseñanza de la cartografía, donde primero se ven los temas asociados a división política y luego los demás mapas temáticos. Además, el lenguaje simbólico de los mapas es un proceso que se inicia en el último ciclo de la educación primaria y se desarrolla en la educación secundaria, el cual al final genera el paso del pensamiento concreto al abstracto (Baquedano, 2014).

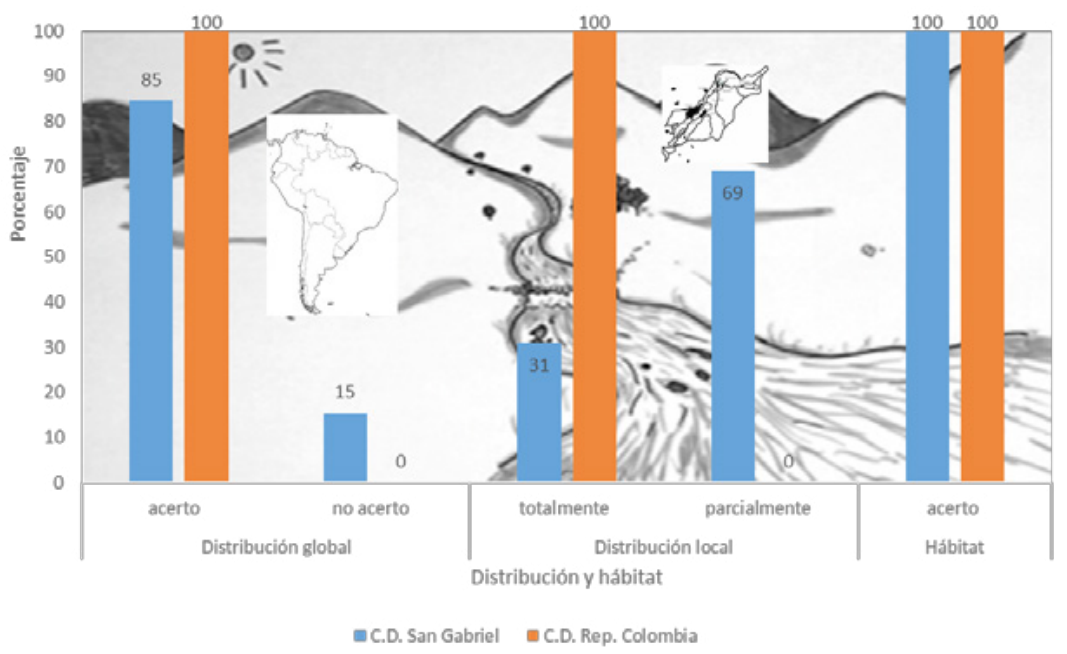

Figura 4. Resultado de la evaluación acerca de los temas de distribución y hábitat del pato de torrente. 
Con relación a la pregunta sobre evaluación del conocimiento del hábitat de la especie, donde se les pidió a los niños que ayudaran a encontrar la casa del pato señalándola con un chulito, todos los niños de los dos centros educativos acertaron (figura 4). Esto significa que se logró llenar el vacío de conocimiento inicial de los niños acerca del hábitat de la especie (ríos cercanos a su casa). Al evaluar la competencia entre la especie y otra especie exótica (trucha arcoíris), se les solicitó a los estudiantes encerrar en un círculo el animal que compite con la especie por alimento. En el C.D. San Gabriel todos los niños acertaron, mientras que en el C.D. República de Colombia acertó el 90 \% (figura 5). Con relación a este tema, es importante que los niños tengan claro que las truchas son una amenaza latente hacia la supervivencia de los patos, pues según Gómez-Zuluaga y Bernal (2013) las truchas compiten fuertemente por alimento con ellos y pueden a futuro desplazarlos. Además, en el municipio de Salento la trucha es una especie exótica invasora imbricada fuertemente en la cultura y la economía del pueblo, lo que dificulta las acciones de conservación de las especies nativas (Botero-Botero y Torres-Mejía, 2010).

$\mathrm{Al}$ evaluar las acciones humanas que ponen en peligro al pato de torrente, se solicitó a los niños señalar con un círculo los dibujos que a criterio de ellos representaban amenazas para el pato de torrente. En el CD San Gabriel el $15 \%$ tuvo dos aciertos, y el restante $85 \%$, tres aciertos. En el CD República de Colombia el $10 \%$ registró cuatro aciertos; el $80 \%$, tres aciertos, y el restante $10 \%$, ningún acierto (figura 5). Dada la importancia de este tema, se decidió realizar el pendón titulado "Los patos de torrentes, aliados en la conservación de nuestros ríos", donde se listan acciones positivas que favorecen a esta especie.

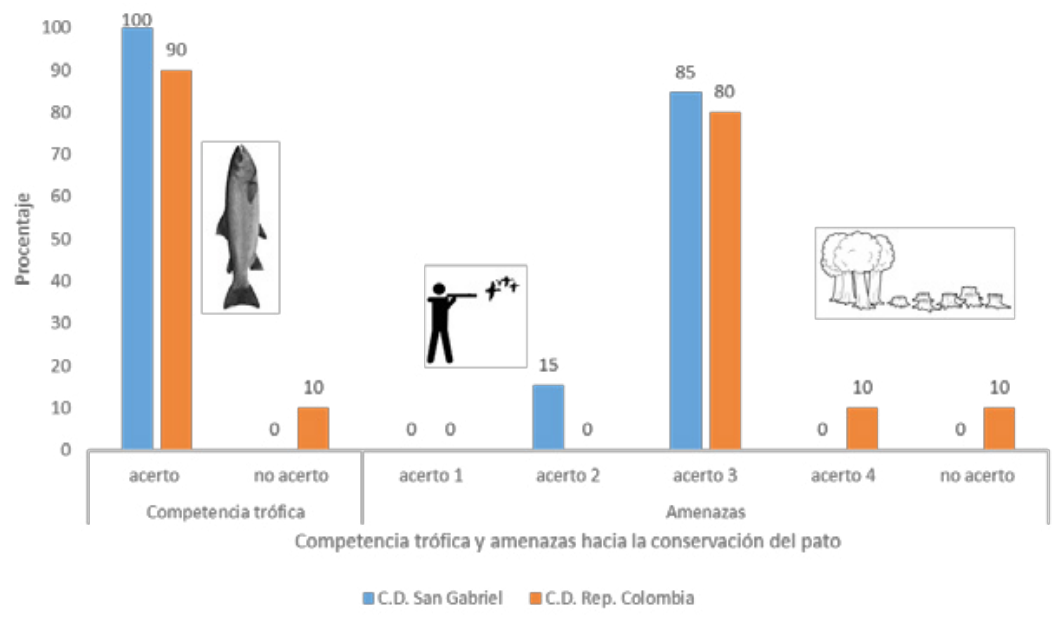

Figura 5. Resultado de la evaluación acerca de los temas de competencia trófica y amenazas humanas para el pato de torrente.

\section{Conclusiones}

La fase de diagnóstico inicial usando el dibujo como herramienta, arrojó que muy pocos niños tenían conocimiento básico sobre el pato de torrente, por lo que fue preciso diseñar una estrategia de intervención para contrarrestar este desconocimiento. Por otro lado, el mismo diseño de la estrategia de educación ambiental basada en estándares curriculares facilitó su aplicación, dado que apoya las labores de aula en el área de las ciencias naturales y suministra al docente material local para su enseñanza.

Se presentó dificultad en la enseñanza del tema dieta del pato de torrente, pues la interacción de los niños 40 con insectos acuáticos es baja en virtud a los hábitats de estos organismos. En efecto, estos macro invertebrados acuáticos no son frecuentemente observados, pues porque se encuentran dentro del cauce del río. En contraste, fue relativamente sencillo manejar el tema de la reproducción de las aves, dados los conocimientos previos de los niños en esta materia. Además, el hecho de ser un asunto ampliamente abarcado en los textos escolares y en las clases de ciencias naturales facilita su comprensión.

Las preguntas que se basaron en procesos memorísticos presentaron el más bajo porcentaje de acierto, lo cual demuestra que con el tiempo los niños van olvidando datos puntuales como el color de las patas o del pico de un ave. A los estudiantes les fue más familiar el mapa de América Latina con la división política que el mapa de 
ríos; esto posiblemente se debe a la forma de enseñanza de la cartografía, donde primero se ven los temas asociados a división política y luego los demás mapas temáticos; asimismo, el lenguaje simbólico de los mapas es un proceso que se inicia en el último ciclo de la educación primaria y es complejo de aprender. En general, los niños comprendieron todos los temas socializados y solo presentaron dificultades en el tema de la dieta de la especie.

\section{Referencias}

Bachelard, G. 1976. La formación del espíritu científico. Siglo Veintiuno Editores S.A., Ciudad de México.

Baquedano, J. 2014. El mapa como instrumento didáctico en la Educación Primaria. Tesis de pregrado, Universidad de Valladolid, Valladolid, España.

Botero-Botero, A., Correa-Viana, M., Torres-Mejía, A., M., Utrera, A. y Kattan G. 2016. Extensión de presencia y área de ocupación de la nutria neotropical (Lontra longicaudis) en la cuenca del río La Vieja, Alto Cauca, Colombia. Boletín Científico Centro de Museos Museo de Historia Natural 20 (1): 101-115.

Botero-Botero, A., Guzmán-Valencia, C., Bernal, K., GómezZuluaga, G., Ríos-Serna, M. y Sánchez-Palma, E. 2015. La lúdica y el juego en la conservación del Pato de torrentes (Merganetta armata, Gould, 1842): una experiencia en el departamento del Quindío, Colombia. Biocenosis 30 (1-2): 28-33.

Botero-Botero, A. y Torres-Mejía, A.M. 2010. Caracterización de la comunidad de pescadores de la cuenca del río La Vieja, Alto Cauca, Colombia. Memorias del III Congreso Colombiano de Zoología, Medellín, Colombia.

Carbonell, M., Kriese, K. y Alexander, K. 2007. Anátidas de la Región Neotropical. Ducks Unlimited INC, Memphis,

Carboneras, C. 1992. Family: Anatidae. En: Del Hoyo, J., Elliot, A. y Sargatal, J. Editor. Handbook of the birds of the World. Vol 1. Lynx Editions, Barcelona.

Cerón, G., Trejos, A. y Kun, M. 2010. Feeding Habits of Torrent Ducks (Merganetta armata armata) in Nahuel Huapi National Park, Argentina. Waterbirds 33: 228-235.

Corporación Autónoma Regional Del Quindío (CRQ). 2009. Plan de ordenación y manejo ambiental UMC río Quindío.

Costa Neto, E.M., Santos Fita, D. y Vargas-Clavijo, M. 2009. Manual de etnozoología. Una guía teórico-práctica para investigar la interconexión del ser humano con los animales. Tundra Ediciones, Valencia.

Fjeldså, J. y Krabbe, N. 1990. Birds of the high Andes: A Manual to the Birds of the temperate zone of the Andes and Patagonia, South America. University of Copenhagen, Copenhagen.

Giraldo-López, T. y Botero-Botero, A. 2017. Efecto de la presencia humana sobre el comportamiento del pato de torrentes Merganetta armata (Anatidae) en el río Quindío, Boquía, Salento. Revista Interamericana de Ambiente y Turismo - RIAT 13 (2): 194-205.

Gómez-Zuluaga, G. y Bernal, K. 2013. Interacciones tróficas entre la sardina de cola roja (Carlastyanax aurocaudatus), el pato de torrentes (Merganetta armata) y la trucha arcoíris (Oncorhynchus mykiss) en la cuenca media-alta del río Quindío. Tesis de pregrado, Universidad del Quindío, Armenia, Colombia.

Hilty, S.L. y Brown, W.L. 2001. Patos, Gansos: Anatidae. En: Hilty, S.L. y Brown, W.L. Editores. Guía de las aves de Colombia. Asociación Colombiana de Ornitología, Colombia.

Madge, S. y Burn, H. 1988. Wildfowl. Christopher Helm, Londres.

Ministerio de Educación Nacional de Colombia, 2008. Estándares Básicos de Competencias en Ciencias Naturales y Ciencias Sociales. Ministerio de Educación Nacional de Colombia, Bogotá.

Ministerio de Educación Nacional de Colombia, 2010. Escuela Nueva. Manual de implementación Escuela Nueva Generalidades y Orientaciones Pedagógicas para Transición y Primer Grado, Tomo I. Ministerio de Educación Nacional de Colombia, Bogotá.

Moffett, G.M. 1970. A study of nesting Torrent Ducks in the Andes. Living Bird 9:5-27.

Naranjo, L. y Ávila, V. 2003. Distribución habitacional y dieta del pato de torrentes (Merganetta armata) en el parque regional Ucumarí en la cordillera central de Colombia. Ornitología Colombiana 1:22-28.

Ramírez U., L.M., Botero, B.Á. y Kattan, G. 2014. Distribución y abundancia del pato de torrentes Merganetta armata (Aves: Anatidae) en el río Quindío (Colombia). Boletín Científico del Centro de museos de la Universidad de Caldas 18(2):172-180.

Robles-Piñeros, J. 2013. Los insectos como estrategia didáctica en la enseñanza de la ecología, a través del cómic. Bio-grafía 6 (10):11-21. 
Ruíz, O.F. 2007. Modelos didácticos para la enseñanza de las ciencias naturales. Revista Latinoamericana de Estudios Educativos (Colombia) 3(2):41-60.

Santos-Baptista, G.C. 2009. Etnozoología y la enseñanza de Villar, R. 1996. El programa Escuela Nueva en Colombia.

las ciencias biológicas. En: Costa Neto, E. M., Santos Fita, D. Revista Educación y Pedagogía 14-15:357-382. y Vargas Clavijo, M. Editores. Manual de etnozoología. Una guía teórico-práctica para investigar la interconexión del ser humano con los animales. Tundra Ediciones, Valencia.

Citar como: López-Londoño, J.E. y Botero-Botero, A. 2019. Estrategias de educación ambiental para promover la conservación del pato de torrente Merganeta armatta colombiana en dos instituciones educativas rurales del municipio de Salento, Quindío, Colombia. Intropica 14(1): 34-42. DOI: http://dx.doi.org/10.21676/23897864.2977 